\title{
Pseudoexfoliation Glaucoma in Greece
}

\author{
A. G. KONSTAS* AND D. ALLAN** \\ Glasgow
}

\begin{abstract}
Summary
Pseudoexfoliation glaucoma is commonly seen in Greece, however there is little data concerning the prevalence and characteristics of this disorder. Patients undergoing trabeculectomy for open angle glaucoma were investigated both by the appraisal of the case notes and by re-examination. The prevalence of pseudoexfoliation glaucoma in this population was found to be $87.8 \%$. The characteristics of this disease process in the population studied are discussed. These findings indicate that pseudoexfoliation is a major contributor to severe glaucoma in the population of Northern Greece.
\end{abstract}

Pseudoexfoliation glaucoma (PXG) is a form of secondary open angle glaucoma which may develop in the elderly as a consequence of the pseudoexfoliation syndrome. ${ }^{1,2}$ This syndrome is characterised by the production and deposition of an abnormal material which is, as yet, of unknown origin and make-up..$^{3-5}$ It may be found throughout the anterior segment of the eye.$^{6-9}$ PXG exhibits a number of clinical characteristics which differ from those of primary open angle glaucoma (POAG). These features include atrophy of the pupillary margin, ${ }^{10}$ iris transillumination defects, ${ }^{11}$ loose pigment in the anterior chamber after pupillary dilatation, ${ }^{12}$ pigment deposition upon the corneal endothelium and within the angle, ${ }^{13}$ abnormal permeability and neovascularisation of the iris vasculature ${ }^{14-16}$ and lack of a hypertensive response to steroids. ${ }^{17,18} \mathrm{~A}$ higher incidence of lens opacities has been described ${ }^{19}$ and the complication rate for both intra-capsular and extra-capsular cataract extraction is greater than that for normal eyes. ${ }^{20,21}$ This has been attributed to weakness of the zonules and the presence of iridocapsular adhesions.

PXG has been reported to carry a worse prognosis than other forms of primary open angle glaucoma..$^{22,23}$ Medical treatment usually produces a significant hypotensive response but this may fail quickly. ${ }^{24,25}$ Argon laser trabeculoplasty (ALT) gives rise to a greater initial pressure lowering effect than with POAG.$^{26}$ However, the long term hypotensive effect of this treatment is often not sustained. ${ }^{27}$ Recourse to surgery is often made and is commonly effective in reducing intraocular pressure (IOP) but the effect of surgery upon the preservation of visual function has yet to be studied prospectively.

PXG was first described in Scandinavian patients, but it has now been shown that people of many races may develop the disorder. ${ }^{28,29}$ Recent research has shown that PXG is more common that had hitherto been reported. ${ }^{30}$ The factors which have influenced the reported results include the techniques of examination chosen, the selection criteria for

From: ${ }^{1}$ Tennent Institute of Ophthalmology, Western Infirmary, Glasgow and ${ }^{2}$ Department of Clinical Physics and Bio-engineering, Glasgow.

Correspondence to: Dr. A. G. Konstas, Tennent Institute of Ophthalmology, Western Infirmary, Church Street, Glasgow, G11 6NT. 
the populations studied and the opthalmologists interest. A recent prospective study in the United States has reported the prevalence of PXG in a glaucoma population to be $6 \% .{ }^{31}$ This compares with $50-62 \%$ for Scandinavia. ${ }^{29}$ This disorder is thus subject to significant geographic variation. The comparable figures which have been reported for Great Britain and Ireland vary considerably, being $12.5 \%$ in England, ${ }^{32} 5 \%$ in Scotland ${ }^{33}$ and $63 \%$ in Southern Ireland. ${ }^{34}$ In Greece the percentages quoted range between 40 and $50 .{ }^{35}$ It must be recognised, however, that the true prevalence of the disease remains uncertain owing to the subtlety of the clinical signs in many cases. An internationally accepted grading scheme would diminish the current confusion.

The distribution of PXG in Greek patients for whom surgery has been deemed necessary has not hitherto been reported. Moreover,

Table I The information obtained for each patient

Data obtained from case records

1 Age

2 Visual acuity*

3 IOP mean value of measurements at admission whilst receiving treatment.*

4. Presence of pseudoexfoliation. ${ }^{*}$

5. Characteristics of the lens.*

6. Cup-disc ratio and/or visual field state.

7. Intra/post operative complications.

8. Duration of hospitalisation.

Data sought by clinical examination

1. Visual acuity.*

2. Assessment of bleb-(cystic or diffuse; size)

3. Intra-ocular pressure on follow-up (with or without treatment). ${ }^{*}$

4. Slit lamp examination: for exfoliation within the pupil or upon the lens (with dilatation of the pupil if material not evident). Vitreous face assessment (in 12 patients who had had subsequent intracapsular cataract extraction). Assessment of the inferior iris, corneal endothelim.*

5. Gonioscopy: for the presence of Sampaolesi's line, grading of the degree of pigmentation within the angle, determination of the presence of peripheral anterior synaethiae, exfoliation or dysgenesis, assessment of the depth of the angle.*

6. Cycloscopy. Examination of the ciliary processes and zonules through the peripheral iridectomy (operated eyes).

7. Assessment of the lens recording evidence of anterior capsule dehiscence of phakodonesis.*

8. Cup/disc ratio evaluation with stereoscopic observation.

* Data sought from both eyes. the disease has not yet been comprehensively characterised in this population. We have set out to determine the prevalence of PXG and to delineate the characteristics of this disease process in this group.

\section{Patients and Methods}

The group studied was identified from the surgical records of AHEPA University Hospital, Thessaloniki in Northern Greece. One-hundred and eight phakic patients were identified who had undergone trabeculectomy, for the first time, for uncontrolled open angle glaucoma. (The indications for trabeculectomy in this unit are uncontrolled intraocular pressure or progressive visual field loss despite two or three topical hypotensive agents.) The patients were requested to attend for a follow up examination. Seventy-four patients (90 eyes) who had undergone a trabeculectomy between 1979 and 1988 were examined. Letters were returned from 20 patients, nine were deceased and four were unable to attend. One patient had undergone enucleation of the treated eye.

For cases with bilateral pseudoexfoliation glaucoma the data presented appertains to the 'poorer eye'. As there is a propensity to the development of bilateral disease this strategy was chosen to produce bias towards the worse case condition. Consideration of 'eyes' has been avoided to preclude favourable bias which would otherwise occur by combining such paired dependent variables.

Patients were diagnosed at the time of follow-up as having PXG if they exhibited one or more of the clinical signs of the index eye shown in Table II.

Table II Details of the clinical signs required to make a diagnosis of pseudoexfoliation glaucoma or pseudoexfoliation syndrome

I Observation of pseudoexfoliative material by slit lamp examination with pupillary dilatation when material not observed within the pupillary aperture.

II Presence of Sampaelesi's line on gonioscopy, together with black pigmentation of the trabecular meshwork. (The diagnosis was only made when Sampaolesi's line was present in its complete form).

III 'Frosting' of the ciliary processes or zonules (as seen by cycloscopy through the peripheral iridectomy) with pseudoexfoliative material. 
The same policy was adopted for the fellow eye. In the absence of glaucoma the diagnosis of pseudoexfoliation syndrome was made if any of the criteria I-III were fulfilled.

Table I summarises the data obtained from the case records and the clinical features sought by clinical examination

\section{Results}

Sixty-five patients $(87.8 \%)$ were found to have evidence of PXG (78 eyes).

\section{Population Characteristics}

The average age of the patients with PXG was 65.9 years (range $49-81$ years) at the time of operation. The sex distribution was 18 female to 47 male, giving a ratio of $1: 2.6$. The mean follow-up time was five years and one month (range: one to nine years).

The pre-operative IOP had a mean value of $(37 \pm 10) \mathrm{mm} \mathrm{Hg}$ (mean $\pm \mathrm{SD})$. The final mean IOP was $17 \pm 6 \mathrm{~mm} \mathrm{Hg}$ and the average reduction was $20 \pm 11 \mathrm{~mm} \mathrm{Hg}$ or $54 \%$ (Fig. 1).

Figure 2 illustrates the cup/disc ratio values seen both pre-operatively and at the time of the examination (this data is based on 54 patients as lens opacities precluded adequate visualisation of the optic disc in the remainder.)

At the time of examination 17 patients (26.1\%) had been prescribed medical treatment but two were not using it. This treatment comprised beta blockers in nine cases and pilocarpine combined with beta blockers in eight cases. Twelve patients had undergone a cataract extraction subsequent to trabeculectomy.

\section{Clinical Characteristics}

Sixty-five of the 74 patients examined fulfilled the diagnostic criteria for PXG giving a prevalence of $87.8 \%$ (Table III). In this Table group A (46 patients) is the group for whom the diagnosis of PXG had been made prior to reassessment. The remaining 19 patients were found to have PXG only at the time of re-evaluation.

In three aphakic patients pseudoexfoliative material could not be seen by slit-lamp examination.

Lens opacities sufficient to diminish visual acuity to $6 / 18$ or worse were present in $55.3 \%$ of $\mathrm{PXG}$ patients pre-operatively and in $59.8 \%$ at the time of follow-up. Figures $3 a$ and $3 b$ illustrate the proportions of patients in whom lens opacities were identified.

The presence of such cataract precludes meaningful interpretation of visual acuity of visual field data as indices of satisfactory outcome. The degree of reduction of intra-ocular pressure and the cup-disc ratios were therefore employed to provide measures of 'control' of glaucoma in this group of patients.

The current status of the other ('better') eye was also determined. In 19 cases the fellow eye was described as normal in the case records. Out of those, 16 were found to exhibit signs of pseudoexfoliation at the time of re-examination. In total only nine patients $(13.8 \%)$ exhibited unilateral disease. Dehiscense of the anterior capsule of the lens in association with cataract and phakadonesis have been described as concomitant feature of pseudoexfoliation. ${ }^{35,36}$ In the present series these signs were observed in four cases and two cases respectively.

\section{Discussion}

The results of the present study indicate that pseudoexfoliation is a common cause of glaucoma in those eyes coming to surgery in

\section{Reduction of Intraocular Pressure}

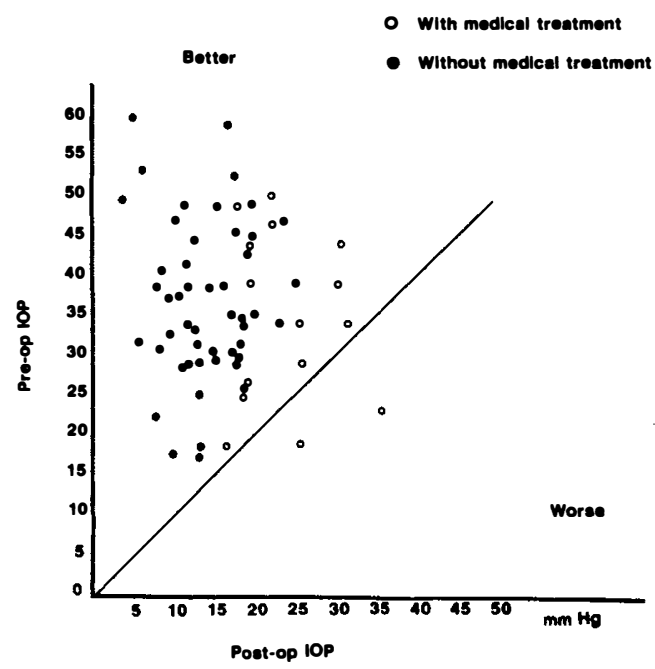

Fig. 1. Pre-operative and post-operative intraocular pressure following trabeculectomy for pseudoexfoliation glaucoma. 

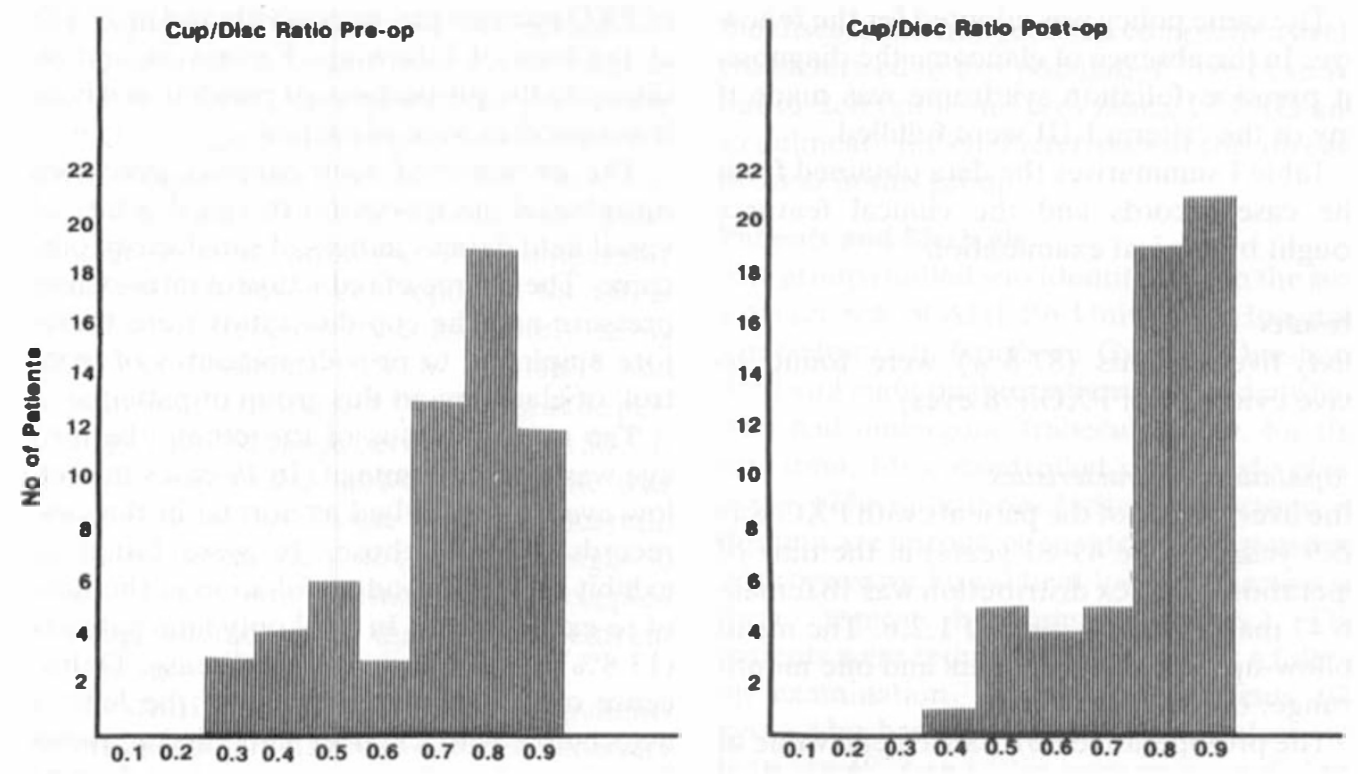

Fig. 2. Cup to disc ratios for 54 patients before trabeculectomy and at the time of reassessment.

Greece. As outlined in the introduction, pseudoexfoliation gives rise to a relatively severe form of chronic glaucoma with high intra-ocular pressures which are less amenable to medical and laser treatment than other forms of chronic glaucoma. Thus the selection criterion of surgical trabeculectomy produces an apparently higher prevalence for the disease than might be expected for the glaucoma population as a whole. This accords with the findings in a number of previous reports. $^{22,32} 87.8 \%$ of patients in this series undergoing trabeculectomy were found to show evidence of pseudoexfoliation in the operated eye. This indicates that pseudoexfoliation is probably responsible for the majority of severe cases of glaucoma in Northern Greece.

The high proportion of cases of PXG identified is markedly in excess of the previously reported figures for comparable surgical populations. ${ }^{22,32}$ If the present study had been conducted as a retrospective analysis only the $62 \%$ of patients would have been identified as having PXG. Additional slit lamp examination only increases the percentage to $71.6 \%$

Table III The positive diagnostic criteria present in 65 patients found to have clinical evidence of pseudoexfoliation glaucoma. Group A comprise those patients for whom the diagnosis had been made at the time of surgery and Group B constitutes those individuals for whom the diagnosis was made at the time of reassessment

Positive diagnostic criteria

\begin{tabular}{lccc}
\hline Patients & I Slit lamp Examination & II Gonioscopy & III Cycloscopy \\
\hline Group A & $43^{*}$ & 45 & 41 \\
46 Patients & & $97.8 \%$ & $91.3 \%$ \\
(10 Aphakics) & $7 * *$ & 19 & 15 \\
Group B & & $100 \%$ & $78.9 \%$ \\
19 Patients & 2 Aphakics) & & \\
\hline
\end{tabular}

\footnotetext{
* Includes seven aphakic patients with pseudoexfoliation on the anterior hyaloid membrane (3) corneal endothelium (2) and pupillary margin only (2).

** Includes one aphakic patient with pseudoexfoliation on the corneal endothelium and pupillary margin.

In three aphakic patients pseudoexfoliative material could not be seen by slit-lamp examination.
} 
PREVALENCE OF CATARACT PREOPERATIVELY

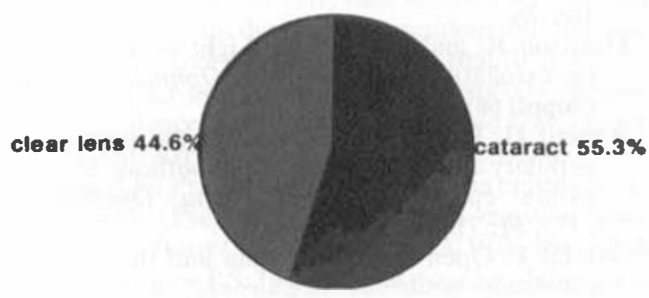

PREVALENCE OF CATARACT POSTOPERATIVELY

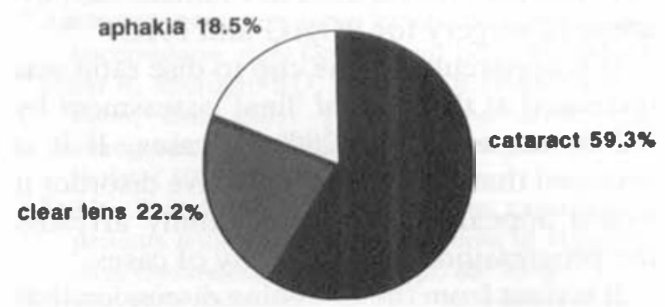

Fig. 3. Distribution of cataract reducing acuity to $6 / 18$ or less, in patients with pseudoexfoliation glaucoma before trabeculectomy and at the time of reassessment.

whereas detailed gonioscopic evaluation in conjunction with cycloscopy was required to produce the figure of $87.8 \%$. Interpretation of retrospective reports in which re-evaluation is not carried out must therefore be made with caution.

When clinical examination is carried out with the intention of identifying pseudoexfoliative material this has been identified in many cases in whom the diagnosis had not hitherto been made. This is particularly true when examination of the contralateral eye is made, in the patient with apparent unilateral pseudoexfoliative glaucoma, employing special techniques such as cycloscopy ${ }^{37}$ and conjunctival biopsy. ${ }^{38}$ Thus the sensitivity for detection of this material by clinical examination varies considerably from centre to centre and is dependent both upon the techniques employed and the interest of the clinicians carrying out the examination. Pseudoexfoliation may therefore be a much commoner cause of sight threatening glaucoma that has previously been thought. This is borne out by the finding that pseudoexfoliative material is commonly identified in eyes with absolute glaucoma and eyes enucleated for rubeotic or painful glaucoma. ${ }^{32,39}$

With regard to clinical assessment of the condition the technique of cycloscopy has only rarely been reported. ${ }^{37}$ In the present series 56 out of 65 patients had positive findings. In all of these cases pseudoexfoliative material was present on the surface of the ciliary processes. However, only in $50 \%$ of those cases the material was present on the surface of the zonules. It is clear from these findings that examination through the peripheral iridectomy can thus be used retrospectively to establish a diagnosis of pseudoexfoliation glaucoma in operated eyes.

The male to female ratio was surprisingly high in our study (2.6). In a number of reports females are more commonly affected than males. ${ }^{40,41}$ While in a Hungarian series the ratio was 1.67 in an inpatient population, ${ }^{42} \mathrm{a}$ report from Australia quotes an even higher ratio (3.0) ${ }^{43}$ In addition an Irish study reports males as having a significantly higher intraocular pressure for each degree of pseudoexfoliation than females. ${ }^{34}$

The reported proportions of bilaterality to unilaterality for PXG also vary considerably. In the present study the prevalence of bilaterality is very high. ( $86.2 \%)$ This may either reflect the advanced state of the disease in the population studied or may represent underdiagnosis in previous reports. This finding highlights the necessity for vigilance in those cases for whom a diagnosis of unilateral disease has been made.

The $55.3 \%$ prevalence of lens opacities in our material correlates well with the results of a current ongoing study in the same Department in which the incidence of the pseudoexfoliation in patients with age related cataract is currently being assessed. So far, more than $50 \%$ of the patients examined exhibit pseudoexfoliative changes. These figures are markedly in excess of the reported prevalence of cataract with the disorder, in Scotland $(2.5 \%),{ }^{33}$ in the United States 
$(20 \%),{ }^{19}$ in Australia $(20 \%)^{43}$ and in Finland $(33 \%) .{ }^{44}$ However, the findings are in accordance with a series reported from Southern Ireland $(51-64 \%) .{ }^{34}$ The presence of cataract makes it difficult to draw comparisons between the criteria used to evaluate the outcome of surgery for POAG and PXG. ${ }^{45,46}$

It is apparent that the cup to disc ratio was increased at the time of 'final' assessment by 0.2 or more in only $20 \%$ of cases. If it is assumed that $\mathrm{PXG}$ is a progressive disorder it would appear that trabeculectomy arrested the progression in the majority of cases.

It is clear from the foregoing discussion that the geographic distribution of the disease varies considerably. Moreover, the findings which we report suggest that there may also be geographic variation in the clinical characteristics of this disorder, a phenomenon which remains to be explained.

Pseudoexfoliation is clearly a major cause of blindness worldwide. The pathogenesis of this disease remains an enigma and further studies are required to delineate both the cause of the disease and its contribution to blindness worldwide.

We are indebted to Gordon N Dutton for all his helpful comments and we wish to thank Sophia Cameron and Dorothy Aitken for their help with the graphs, Lorna Brown for typing the manuscript and all the staff of the Eye Department, AHEPA Hospital, Thessaloniki, Greece.

\section{References}

${ }^{1}$ Aasved H: Relationship of intraocular pressure and fibrillopathia epitheliocapsularis. Trans Ophthalmol Soc UK 1979, 99: 310-1.

${ }^{2}$ Sugar HS: Pigmentary glaucoma and the glaucoma associated with the exfoliation-pseudoexfoliation syndrome: Update. Ophthalmology 1984, 91: 307-10.

${ }^{3}$ Ghosh $\mathrm{H}$ and Speakman JS: The origin of senile lens exfoliation. Can J Ophthalmol 1983, 18: 340-3.

${ }^{4}$ Ringvold $\mathrm{A}$ and Davanger $\mathrm{M}$ : Notes on the distribution of pseudo-exfoliation material with particular reference to the uveoscleral route of aqueous humour. Acta Ophthalmol 1977, 55: 807-14.

${ }^{5}$ Sugar HS: The exfoliation syndrome: Source of the fibrillar material in the capsule. Surv Ophthalmol 1976, 21: 59-64.

${ }^{6}$ Eagle RC, Font RL, Fine BS: The basement membrane exfoliation syndrome. Arch Ophthalmol 1979, 97: 510-5.

${ }^{7}$ Ringvold A: On the occurence of pseudo-exfoliation material in extrabulbar tissue from patients with pseudo-exfoliation syndrome of the eye. Acta Ophthalmol 1973, 51: 411-8.

${ }^{8}$ Ringvold A: The distribution of the exfoliation material in the iris from eyes with exfoliation syndrome (Pseudoexfoliation of the lens capsule). Virchows Arch Abt A Path Anat 1970, 351: 168-78.

${ }^{9}$ Morrison JC and Green WR: Light microscopy of the exfoliation syndrome. Acta Ophthalmol 1988, (Suppl) 66: 5-27.

${ }^{10}$ Aasved H: Incidence of defects in the pigmented pupillary ruff in eyes with and without fibrillopathia epitheliocapsularis. Acta Ophthalmol 1973, 51: 710-5.

${ }^{11}$ Jerndal T: Open angle glaucoma and the pseudoexfoliation syndrome. In Cairns JE ed Glaucomas London: Grune and Stratton 1987, 661-77.

${ }^{12}$ Krause U, Helve J, Forcius H: Pseudoexfoliation of the lens capsule and liberation of iris pigment. Acta Ophthalmol 1973, 51: 39-46.

${ }^{13}$ Sampaolesi R, Zarate J, Croxato O: The chamber angle in exfoliation syndrome. Acta Ophthalmol 1988, (Suppl) 66: 48-53.

${ }^{14}$ Vannas A: Fluorescein angiography of the vessels of the iris in pseudoexfoliation of the lens capsule, capsular glaucoma and some other forms of glaucoma. Acta Ophthalmol 1969, (Suppl) 105: 9-75.

${ }^{15}$ Ringvold $\mathrm{A}$ and Davanger M: Iris neovascularisation in eyes with pseudoexfoliation syndrome. $\mathrm{Br}$ J Ophthalmol 1981, 65: 138-41.

${ }^{16}$ Brooks AM and Gillies WE: The development of microvascular changes in the iris in pseudoexfoliation of the lens capsule. Ophthalmology 1987, 94: 1090-7.

${ }^{17}$ Pohjola S and Horsmanheimo A: Topically applied corticosteroids in glaucoma capsulare. Arch Ophthalmol 1971, 85: 150-3.

${ }^{18}$ Gillies WE: Corticosteroid induced ocular hypertension in pseudo-exfoliation of the lens capsule. Am J Ophthalmol 1970, 70: 90-5.

${ }^{19}$ Layden WE and Shaffer RN: Exfoliation syndrome. Am J Ophthalmol 1974, 78: 835-41.

${ }^{20}$ Dark AJ. Cataract extraction complicated by capsular glaucoma. Br J Ophthalmol 1979, 63: 465-8.

${ }^{21}$ Raitta C and Setala K: Intraocular lens implantation in exfoliation syndrome and capsular glaucoma. Acta Ophthalmol 1986, 64: 130-3.

${ }^{22}$ Tarkkanen AH: Exfoliation syndrome. Trans Ophthalmol Soc UK 1986, 105: 233-6.

${ }^{23}$ Olivius E and Thorburn W: Prognosis of glaucoma simplex and glaucoma capsulare. A comparative study. Acta Ophthalmol 1978, 56: 921-34.

${ }^{24}$ Blika S and Saunte E: Timolol maleate in the treatment of glaucoma simplex and glaucoma cassulare. Acta Ophthalmol 1982, 60: 967-76.

${ }^{25}$ Tarkkanen AH: Treatment of chronic open-angle glaucoma associated with pseudoexfoliation. Acta Ophthalmol 1965, 43: 514-23.

${ }^{26}$ Tuulonen A, Airaksinen PJ, Kuulasmaa K: Factors influencing the outcome of laser trabeculoplasty. Am J Ophthalmol 1985, 99: 388-91.

${ }^{27}$ Svedbergh B: Argon laser trabeculoplasty in capsular glaucoma. Acta Ophthalmol 1988, (Suppl) 66: 141-7.

${ }^{28}$ Forsius H: Prevalence of pseudoexfoliation of the 
lens in Finns, Lapps, Icelanders, Eskimos and Russians. Trans Ophthalmol Soc UK 1979, 99: 296-8.

${ }^{29}$ Forsius $\mathrm{H}$ : Exfoliation syndrome in various ethnic populations. Acta Ophthalmol 1988, (Suppl) 66: 71-85.

${ }^{30}$ Prince AM, Streeten BW, Rich R, Dark AJ, Sperling M: Preclinical diagnosis of pseudoexfoliation syndrome. Arch Ophthalmol 1987, 105: 1076-82.

${ }^{31}$ Cash well LF and Shields MB: Exfoliation syndrome. Prevalence in a Southeastern United States population. Arch Ophthalmol 1988, 106: 335-6.

${ }^{32}$ Aasved H: Prevalence of fibrillopathia epitheliocapsularis (Pseudo-exfoliation) and capsular glaucoma. Trans Ophthalmol Soc UK 1979, 99: 293-5.

${ }^{33}$ Bartholomew RS: Incidence of pseudoexfoliation in South African Negroes and Scots. Trans Ophthalmol Soc UK 1979, 99: 299-301.

${ }^{34}$ Madden JG and Crowley MJ: Factors in the exfoliation syndrome. Br J Ophthalmol 1982, 66: 432-7.

${ }^{35}$ Konstas P, Demetracoulias N, Zeseadis D: Pseudoexfoliation glaucoma. 1985 Round table discussion in XXI Congress in Ophthalmology Greece.

${ }^{36}$ Bartholomew RS: Lens displacement associated with pseudocapsular exfoliation. A report on 19 cases in the Southern Bantu. Br J Ophthalmol 1970, 54: 744-50.

${ }^{37}$ Mizuno K and Muroi S: Cycloscopy of pseudoexfoliation. Am J Ophthalmol 1979, 87: 513-8.
${ }^{38}$ Speakman JS and Ghosh M: The conjunctiva in senile lens exfoliation. Arch Ophthalmol 1976, 94: 1757-9.

${ }^{39}$ Karjalainen K, Tarkkanen A, Merenmies L: Exfoliation syndrome in enucleated haemorrhagic and absolute glaucoma. Acta Ophthalmol 1987, 65: 320-2.

${ }^{40}$ Aasved H: Mass screening for fibrillopathia epitheliocapsularis. Acta Ophthalmol 1971, 49: 334-43.

${ }^{41}$ Hiller R, Sperduto RD, Krueger DE: Pseudoexfoliation, intraocular pressure and senile lens changes in a population based survey. Arch Ophthalmol 1982, 100: 1080-2.

${ }^{42}$ Sziklai $P$ and Suveges I: Glaucoma capsulare in patients with open angle glaucoma in Hungary. Acta Ophthalmol 1988, (Suppl) 66: 90-2.

${ }^{43}$ Taylor HR: Pseudoexfoliation, an environmental disease? Trans Ophthalmol Soc UK 1979, 99: $302-6$.

${ }^{44}$ Krause U and Tarkkanen AH: Cataract and pseudoexfoliation. A clinicopathological study. Acta Ophthalmol (Copenh) 1978, 56: 329-34.

${ }^{45}$ Raitta C and Setala K: Argon Laser Trabeculoplasty versus trabeculectomy in simple and capsular glaucoma: A long term follow up. Glaucoma 1986, 8: 141-9.

${ }^{46}$ Jerndal T and Kriisa V: Results of trabeculectomy for pseudoexfoliative glaucoma, a study of 52 cases. Br J Ophthalmol 1974, 58: 927-30. 